\title{
Study on pulsed thermography to detect water ingress in composite honeycomb panels
}

\author{
Xingwang Guo*, Feifei Zhang* \\ * School of Mechanical Engineering and Automation, Beihang University, Beijing 100191, P. R. China, \\ xingwangguo@buaa.edu.cn
}

\begin{abstract}
The pulsed thermography (PT) for water detection in aviation composite honeycomb panels was studied by finite element method and experiment. A 3-D heat conduction model of water detection in honeycomb panels was presented; the relationships between information parameters and water content were analyzed for three testing modes, i.e. the near water testing, far water testing and vertical testing. Some theoretical results were verified by experiment. The results indicate that, in the three testing modes, both the maximum differential temperature and maximum contrast are non-linear functions of water height, and increase with the water height. The detection sensitivity of the near water testing is the highest, the vertical testing takes the second place, and the far water testing is the lowest. The water content can be evaluated inversely by the curve of maximum contrast versus water height. The obtained results provide a technique support for water detection and evaluation of aviation honeycomb structures by PT.
\end{abstract}

\section{Introduction}

The use of honeycomb sandwich structures is widespread on aircrafts. They provide stiff lightweight structures which are ideal for control surfaces and other exterior structures, such as radome, rudders, stabilizers, ailerons, etc. Water ingress in honeycomb structures can degrade epoxy adhesives, erode metal, and add unnecessary weight to the structure. The water in honeycomb cells is a potential destroyer to cells as it expands after freezing. So the nondestructive testing technique for detecting water in honeycomb structures is very important in aircraft maintenance. The traditional techniques used by aircraft maintenance technicians to inspect honeycomb composite material are ultrasonic and X-ray technique. However, the point-by-point ultrasonic inspection is low-productive, while the X-ray technology is hardly used in situ because it requires two-side installation and strict radiation safety precautions. With the success of infrared thermography (IRT) in detecting composite materials [1-3], the IRT for detecting water ingress in honeycomb structures attracts people's attention [4]. IRT is a high-speed, large area, portable, non-contact and safety inspection technique, and can be used in one-side testing, so it can make up the deficiencies of the traditional methods.

In the 1990's, Airbus Industry, Inc. had developed an infrared thermographic inspection technique by using an electrothermal blanket as the active heating source to detect water ingress in composite sandwich structures. Airbus Industry, Inc., recommended that all operators of its aircraft adopt this method as the standard inspection measure for such parts [5-6]. Since 2000, optical excitation became dominant in active IRT, and the IRT for detecting water ingress in honeycomb structures had been developed quickly. The representative work was accomplished by V. Vavilov and his research team. They had investigated the IRT of water hidden in honeycomb panels of aircraft from modeling, experimental analysis and field testing aspect [7-9]. A 2D cylindrical model, which includes several sub-regions corresponding to a honeycomb skin, cells, glue and water, was used to simulate the heat conduction in detecting water in honeycomb cells[7]. The water content was evaluated by using the ice-to-water phase transformation in transient IRT; a 1D model that involves the ice-to-water phase transformation was developed to overcome the discrepancy between the theoretical predictions and practical results [8]. Eetta Saarimakia had also investigated the thermographic inspection exploiting phase transition of water [10]. The whole aircraft structure is cooled below the freezing point of the water and then warmed into the room temperature. Phase transition energy, which is needed to melt the penetrated water, can be detected by thermography. Zhao Shibin had investigated the pulsed thermography (PT) for detecting the presence of water and hydraulic oil within the sandwich panels [11]. The capability of PT is demonstrated through laboratory imaging of both water and hydraulic oil within an aluminum sandwich panels. In conclusion, The NDT of water ingress in honeycomb structures is a very important application of IRT. Although the technique has been studied for many years, the existing results are not enough for industry application. The study on PT of water ingress in honeycomb structures is still not sufficient and systematic. For instance, the quantitative influences of testing approaches, water content, structure parameters and heating parameters on the detection effect are not revealed clearly. So there is still a long way to go.

The aim of this work is to investigate the rules of pulsed thermography (PT) of water ingress in composite honeycomb panels in normal temperature circumstance. A new 3-D simulation model is presented, and the heat transfer of PT for water detection is simulated by finite element method (FEM). The influences of some significant factors, such as the testing approaches, water content, face panel thickness, etc., on the detection effect are quantitatively researched, and some of the theoretical results are validated by experiment. 


\section{Modeling water detection in honeycomb structures}

Usually honeycomb cells are hexagonal. It is not convenient to mesh such structures into elements in FEA. Based on keeping the thickness, height and volume of the total cell wall unchanged, the hexagonal cells were converted into square cells. A region of the simplified honeycomb structure is shown in Fig.1.

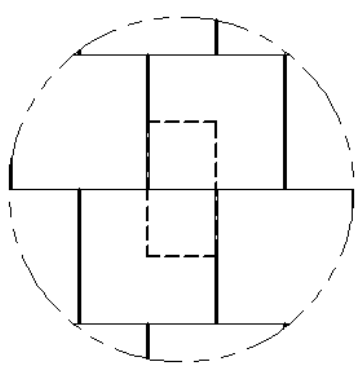

Fig.1. Scheme of the simplified honeycomb structure

The geometric pattern and size of all square cells are consistent. The structure is symmetrical in a local area to the mirrored planes as indicated with the dashed lines in Fig.1. Assuming the heating be planar, no heat transfer through the symmetrical plane. In order to shorten the calculating time, a model which consists of two 1/4 parts of the two adjacent square cells was constructed with four adiabatic boundaries, as shown in Fig.2, where one of the cells partially filled with water. According to the specification of a honeycomb structure part of an aircraft, the material of the face panel is glass fiber reinforced plastic (GFRP), the core is Nomex®.

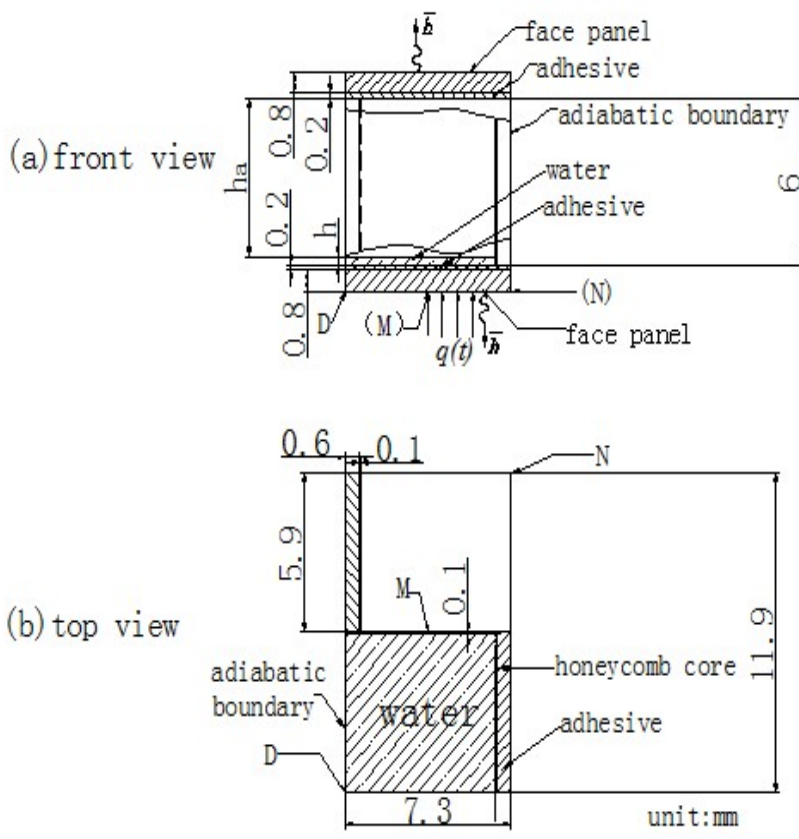

Fig.2. Modeling water detection in honeycomb structures

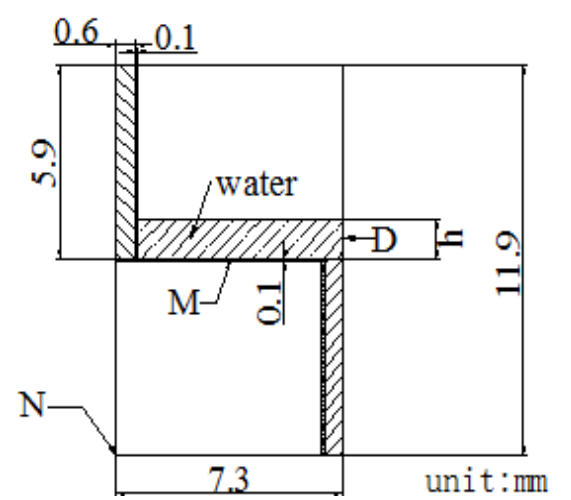

Fig. 3. Showing water area center $D$ and sound area center $N$ as the panel is vertically laid

To simplify the analysis, assume the conductivity of each material is isotropic. Assume the conditions as follows: a convective heat exchange exists on the face panel surfaces; the radiant heat exchange is ignored; the model's side boundary is adiabatic; the initial temperature is uniform and equal to the environment temperature $T_{0}$; the panel is heated by a rectangular pulse whose heat flux density is $q(t)$,

$$
q(t)= \begin{cases}q_{\max } & 0 \leq t \leq t_{\mathrm{d}} \\ 0 & t>t_{\mathrm{d}}\end{cases}
$$

where $q_{\max }$ is the amplitude of the heat flux density, $t_{\mathrm{d}}$ the heating duration. 
The investigated one-side testing separates into three different testing approaches according to the relative position of monitored surface from the water. The first approach is called near water testing, in which the honeycomb panel is horizontally laid and its underside surface near the water is heated and monitored. The second approach is called far water testing, in which the honeycomb panel is horizontally laid and its upper surface apart from the water is heated and monitored. The third approach is called vertical testing, in which the honeycomb panel is vertically placed and one of its surfaces is heated and monitored while the water is gathering on cell walls.

The water content is expressed by a water height $h$ in the near water testing and far water testing. To express the water content in the uniform parameter, the actual water height $h$ in the vertical testing (as shown in Fig.3) needs to be replaced by an equivalent water height $h_{\mathrm{e}}$ as follows:

$$
h_{\mathrm{e}}=\frac{(\text { area of one wal of a cel }) \times h}{\text { base area of a cell }}=0.508 \mathrm{~h}
$$

\section{Simulation method}

\subsection{Information parameters}

The temperature evolution of the surface point $\mathrm{D}$ and $\mathrm{N}$ corresponding to the center of water area and sound area respectively are researched emphatically, and the positions of $D$ and $N$ in the near water testing and vertical testing are shown as Fig. 2 and Fig. 3 respectively. Set the excess temperature at $\mathrm{D}$ and $\mathrm{N}$ are $\theta_{\mathrm{D}}$ and $\theta_{\mathrm{N}}$ respectively, then the differential temperature between water area and sound area can be described by the differential temperature between $\mathrm{D}$ and $\mathrm{N}$ as follows:

$$
\Delta T=\theta_{\mathrm{N}}-\theta_{\mathrm{D}}
$$

where $\Delta T$ is called differential temperature for short

Contrast is defined as:

$$
C=\Delta T / \theta_{\mathrm{N}}
$$

\subsection{Computation strategy based on FEM}

The simulation process is as follow: first of all, calculate the heat transfer of the water detection model (see Fig.2 3) by FEM using the commercial software ANSYS; second, process the result data by a Matlab program to extract the maximum temperature difference, maximum contrast and maximum excess temperature; finally, summarize the laws of how the water height and testing approach influence the information parameters.

The geometrical parameters of the model are shown in Fig.1, where each cell has a base area of 38.94 $\mathrm{mm}^{2}$, Assume the ambient temperature $T_{0}=0^{\circ} \mathrm{C}$, the convective heat transfer coefficient on panel surface $\bar{h}=10 \mathrm{~W} \cdot \mathrm{m}^{-2} \mathrm{~K}^{-1}$, the heating intensity $q_{\max }=6 \times 10^{5} \mathrm{~W} \cdot \mathrm{m}^{-2}$, the heating duration $t_{\mathrm{d}}=0.01 \mathrm{~s}$, and the observation duration in cooling stage 50 s.

The element type Solid70 for thermal analysis was chosen in the finite element analysis. Considering the simulation precision and time, mesh the face panel, adhesive, honeycomb wall, air and water with cubes of size $0.1 \mathrm{~mm}$, the time step $3 \times 10^{-5} \mathrm{~s}$ in heating stage and $5 \times 10^{-5} \mathrm{~s}$ in cooling stage were chosen. The thermal properties of materials used in the model are given in table 1.

Table 1. Thermal properties of materials

\begin{tabular}{cccc}
\hline Material & $\begin{array}{c}\text { Density } \\
\rho / \mathrm{kg} \cdot \mathrm{m}^{-3}\end{array}$ & $\begin{array}{c}\text { Specific heat capacity } \\
\mathrm{C} / \mathrm{J} \cdot \mathrm{kg}^{-1} \cdot \mathrm{K}^{-1}\end{array}$ & $\begin{array}{c}\text { Heat conductivity } \\
\lambda / \mathrm{W} \cdot \mathrm{m}^{-1} \cdot \mathrm{K}^{-1}\end{array}$ \\
\hline GFRP & 1250 & 1200 & 0.3 \\
Adhesive & 1157 & 1700 & 0.2 \\
Nomex® & 72 & 1700 & 0.1035 \\
Air & 1.2 & 1005 & 0.02 \\
Water & 1000 & 4193 & 0.586 \\
\hline
\end{tabular}




\section{Simulation and discussion}

\subsection{Relationships between maximum differential temperature, maximum contrast and water height in near water} testing

Assuming the water height $h$ is $0.1,0.3,0.5$ and $0.7 \mathrm{~mm}$ respectively in the near testing, the obtained maximum differential temperature $\Delta T_{\mathrm{m}}$ versus water height $h$ is shown in Fig.4

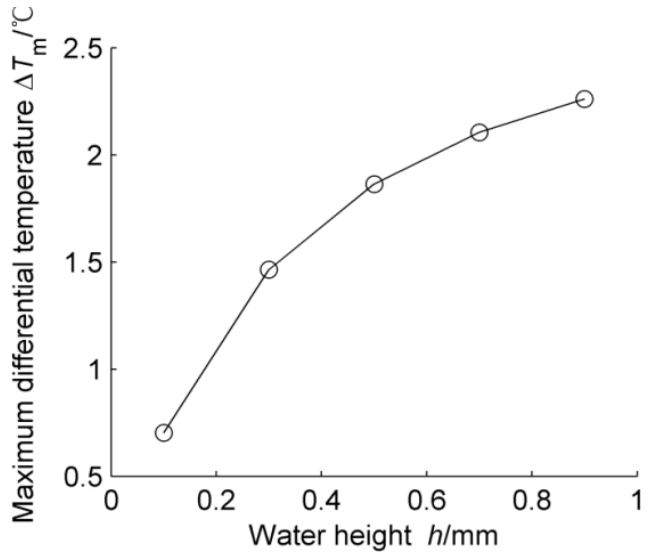

Fig.4. Maximum differential temperature $\Delta T_{\mathrm{m}}$ vs. water height $h$ in near water testing

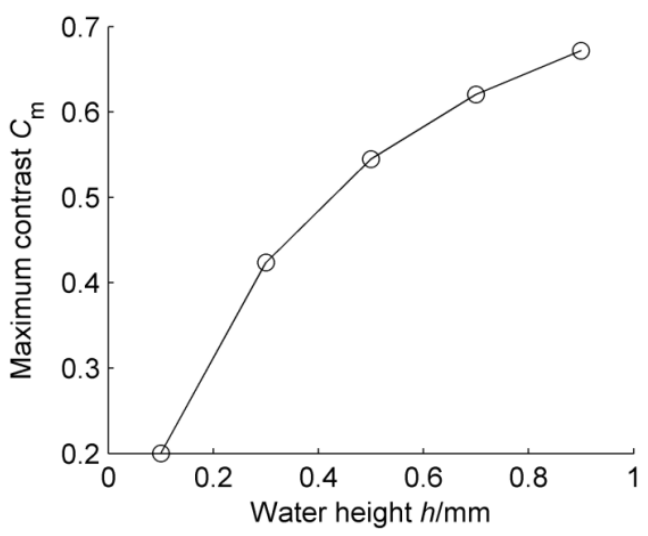

Fig.5. Maximum contrast $C_{\mathrm{m}}$ vs. water height $h$ in near water testing

As it can be seen in Fig.1, the maximum differential temperature increases as the water height increasing, but the growth rate gradually decreases. It can be deduced that the maximum differential temperature will tend to a saturation value when the water height reaches a certain value.

The obtained maximum contrast $C_{\mathrm{m}}$ versus water height $h$ in near water testing is shown in Fig.5, and it shows a similar tendency with that of maximum differential temperature versus water height.

\subsection{Relationships between maximum differential temperature, maximum contrast and water height in far water testing}

Assuming the water height $h$ is $0.1,0.3,0.5,0.7$ and $0.9 \mathrm{~mm}$ respectively in the far water testing, the obtained maximum differential temperature versus water height is shown in Fig.6.

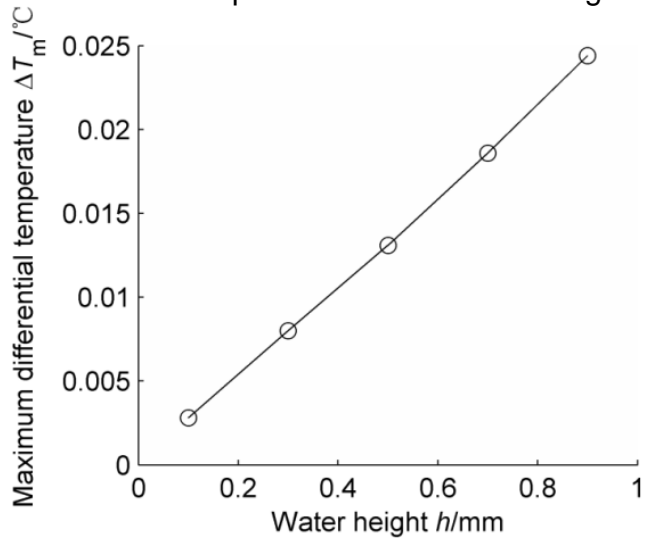

Fig.6. Maximum differential temperature $\Delta T_{\mathrm{m}}$ vs. water height $h$ in far water testing

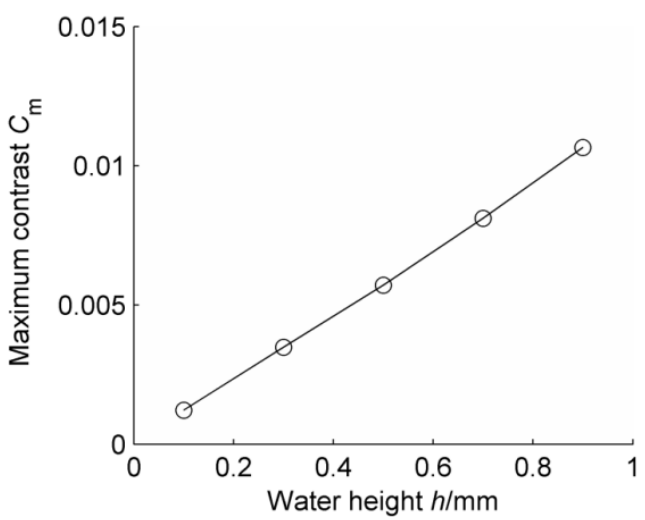

Fig.7. Maximum contrast $C_{\mathrm{m}}$ vs. water height $h$ in far water testing

As it can be seen in Fig.6, the maximum differential temperature cannot reach a distinguishable extent (e.g. $0.1^{\circ} \mathrm{C}$ ) for usual infrared cameras when the water height is in several millimeters although the maximum differential temperature increases as the water height increasing.

The obtained maximum contrast versus water height in far water testing is shown in Fig.7.

In order to explore the detecting limit of the far water testing, assuming the thickness $h_{\mathrm{a}}$ of the air gap between 
the adhesive layer and water is $0.1,0.3,0.5,0.7,0.9$ and $3.4 \mathrm{~mm}$ respectively, the obtained maximum differential temperature versus water height is shown in Fig. 8.

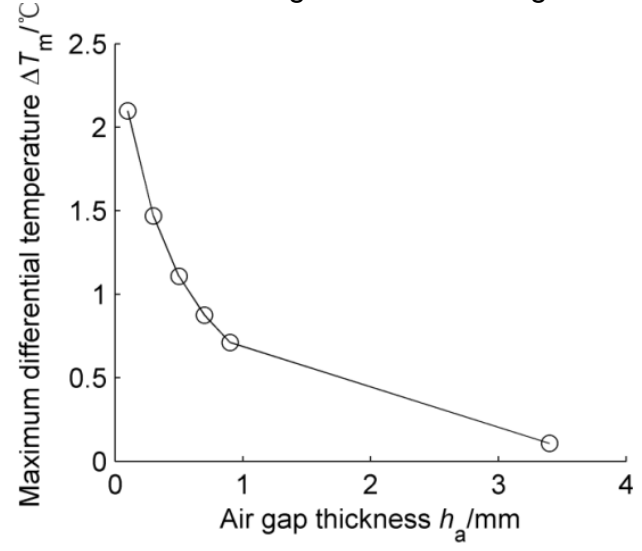

Fig.8. Maximum differential temperature $\Delta T_{\mathrm{m}}$ vs. air gap thickness $h_{\mathrm{a}}$ in far water testing

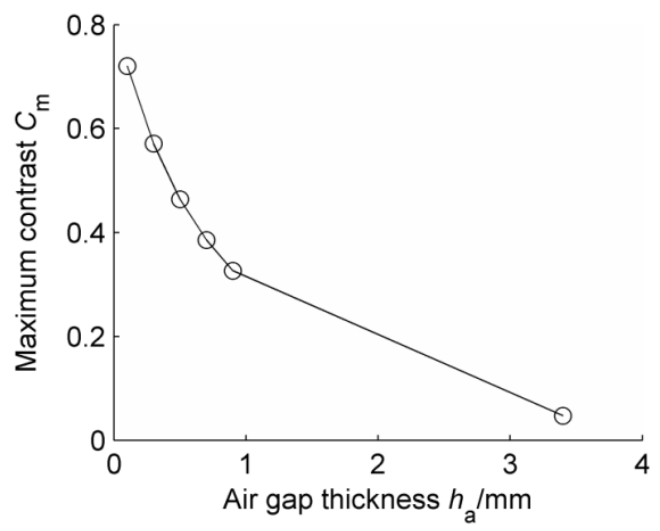

Fig.9. Maximum contrast $C_{\mathrm{m}}$ vs. air gap

thickness $h_{\mathrm{a}}$ in far water testing

It is shown in Fig.8, in far water testing, the maximum differential temperature decreases as the air gap thickness increasing, and the relation curve is non-linear. When the air gap thickness $h_{\mathrm{a}}$ is $3.4 \mathrm{~mm}$, the maximum differential temperature is $0.1^{\circ} \mathrm{C}$ which equals the temperature sensitivity $\left(0.1^{\circ} \mathrm{C}\right)$ of a typical infrared camera, therefore the air gap thickness $3.4 \mathrm{~mm}$ can be taken as the detection limit of far water testing for this honeycomb structure.

The maximum contrast versus air gap thickness in far water testing is shown in Fig.9.

\subsection{Relationships between maximum differential temperature, maximum contrast and equivalent water height in vertical testing}

Assuming the equivalent water height $h_{\mathrm{e}}$ is $0.1,0.3,0.5,0.7$ and $0.9 \mathrm{~mm}$ respectively in the vertical testing, the obtained maximum differential temperature versus equivalent water height is shown in Fig.10, and the maximum contrast versus equivalent water height is shown in Fig.11.

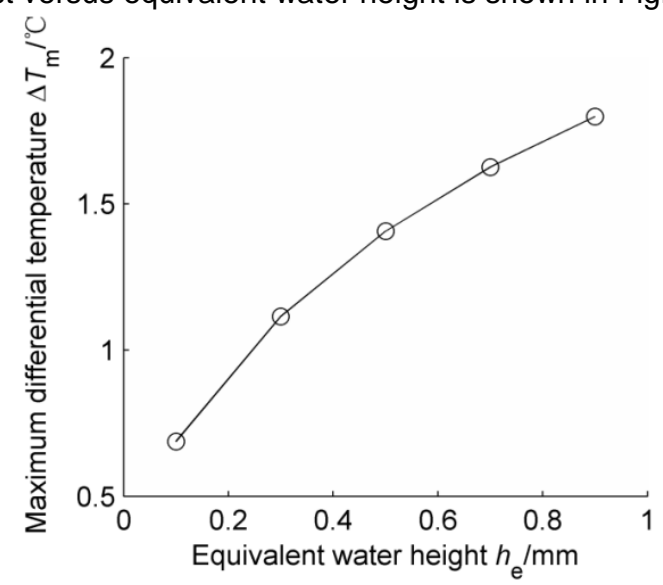

Fig.10. Maximum differential temperature $\Delta T_{\mathrm{m}}$ vs. equivalent water height $h_{\mathrm{e}}$ in vertical testing

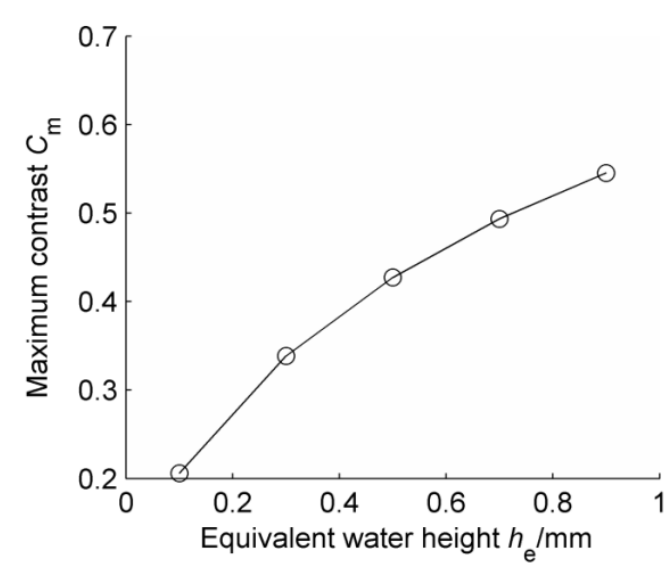

Fig.11. Maximum contrast $C_{\mathrm{m}}$ vs. equivalent water height $h_{\mathrm{e}}$ in vertical testing

\subsection{Influence of face panel thickness on detection}

Among all the structure parameters, face panel thickness plays the most important role in detecting water, so the influence of the face panel thickness on water detection is investigated here. For near water testing, assuming water height $h=0.5 \mathrm{~mm}$, the face panel thickness $h_{\mathrm{f}}$ is $0.4,0.6,0.8,1$ and $1.2 \mathrm{~mm}$ respectively, the other parameters are as above, the obtained maximum differential temperature $\Delta T_{\mathrm{m}}$ versus face panel thickness $h_{\mathrm{f}}$ is shown in Fig.12, 
and the maximum contrast $C_{\mathrm{m}}$ versus face panel thickness $h_{\mathrm{f}}$ is shown in Fig.13.

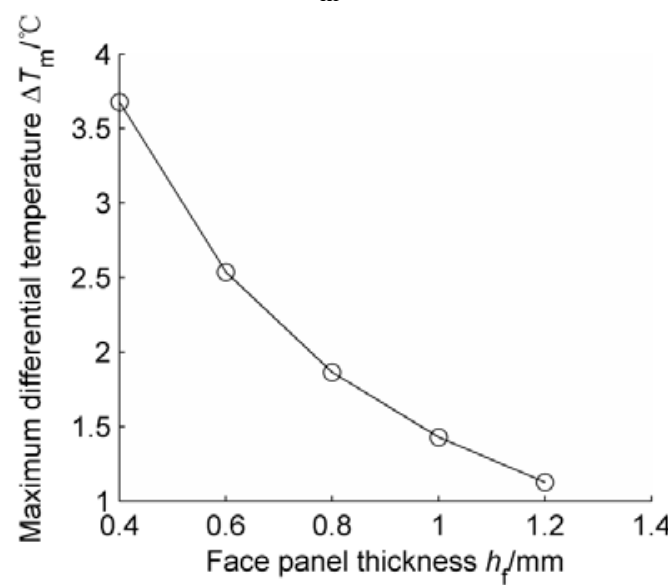

Fig.12. Maximum differential temperature $\Delta T_{\mathrm{m}}$ vs. face panel thickness $h_{\mathrm{f}}$ in near water testing

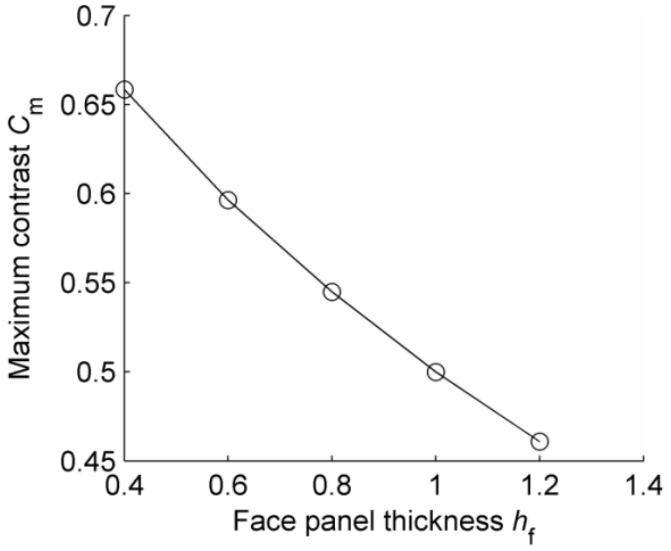

Fig.13. Maximum contrast $C_{\mathrm{m}}$ vs. face panel thickness $h_{\mathrm{f}}$ in near water testing

As it can be seen in Fig.12 and Fig.13, the maximum differential temperature and maximum contrast decrease as the face panel thickness increasing. The maximum differential temperature is inversely proportional to the face panel thickness; the maximum contrast is a linear function of the face panel thickness approximately.

The differential temperature peak time $t_{\mathrm{m}}$ versus surface panel thickness $h_{\mathrm{f}}$ in near water testing is shown in Fig.14.

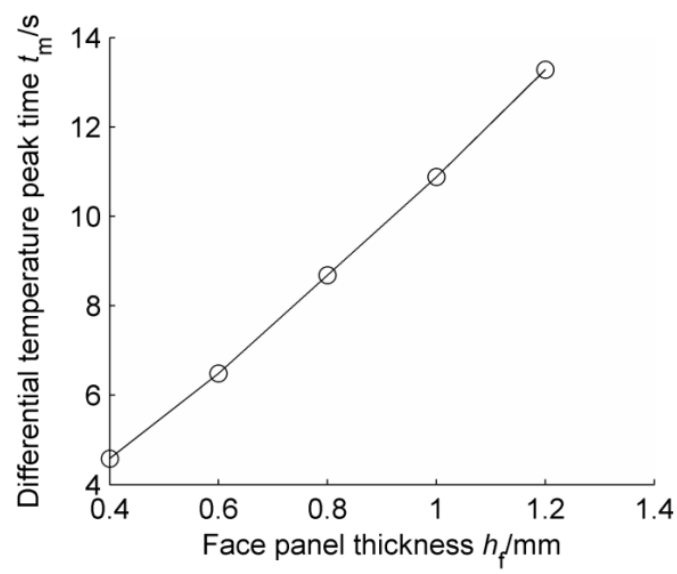

Fig.14. Differential temperature peak time $t_{\mathrm{m}}$ vs. surface panel thickness $h_{\mathrm{f}}$ in near water testing

As it can be seen in Fig.14, the differential temperature peak time is postponed when the face panel thickness increases, and the peak time is a linear function of the face panel thickness approximately. If the differential temperature peak time can be predicted before testing, the thermal image sequence should be captured only in the selected period of time near the peak time, and this will reduce the raw data significantly and it is helpful for large panels.

\section{Experimental analysis}

\subsection{Experimental setup and specimen}

A pulsed thermographic system developed by our lab was used in the experiment. The system consists of an infrared camera, two flash lamps, a heating controller, a computer and an application program. The infrared camera uses an un-cooled detector with a frame rate of $60 \mathrm{~Hz}$, a focal plane array pixel format of $320 \times 240$ and a thermal resolution of $0.1 \mathrm{~K}$. The specimen was heated by the two flash lamps with the total output energy of $6000 \mathrm{~J}$.

The specimen is a composite honeycomb panel with two face panel made of GRFP, a core layer made of Nomex ${ }^{\circledR}$, and two adhesive layers. The thickness of each face panel is $0.7 \mathrm{~mm}$; the thickness of each adhesive layer is $0.1 \mathrm{~mm}$; the height of cells is $6 \mathrm{~mm}$; each cell has a base area of $33.2 \mathrm{~mm}^{2}$. To generate water ingress, nine separated 
cells were chosen, a small hole with a diameter of $0.8 \mathrm{~mm}$ was drilled on the top face panel of each cell, and then the nine cells are filled with quantitative water through injection. The 9 water areas are denominated as No.1, 2, 3, 4, 5, 6, 7, 8, and 9 respectively. The volume of each water area is $1.7,3.3,6.6,10,13.3,16.6,23.2,29.9$ and $36.5 \mathrm{~mm}^{3}$ respectively. The height of each water area is $0.05,0.1,0.2,0.3,0.4,0.5,0.7,0.9$ and $1.1 \mathrm{~mm}$ respectively.

\subsection{Experimental results}

Only the near water testing is analyzed by experiment.

A typical frame of excess temperature image in near water testing of the specimen is shown in Fig.15. The two dark areas in the bottom corners are holders. Fig.15 shows that the water areas No.2 to No.9 can be seen clearly, but the water area No.1 filled with the least water $(h=0.05 \mathrm{~mm})$ is blurry.
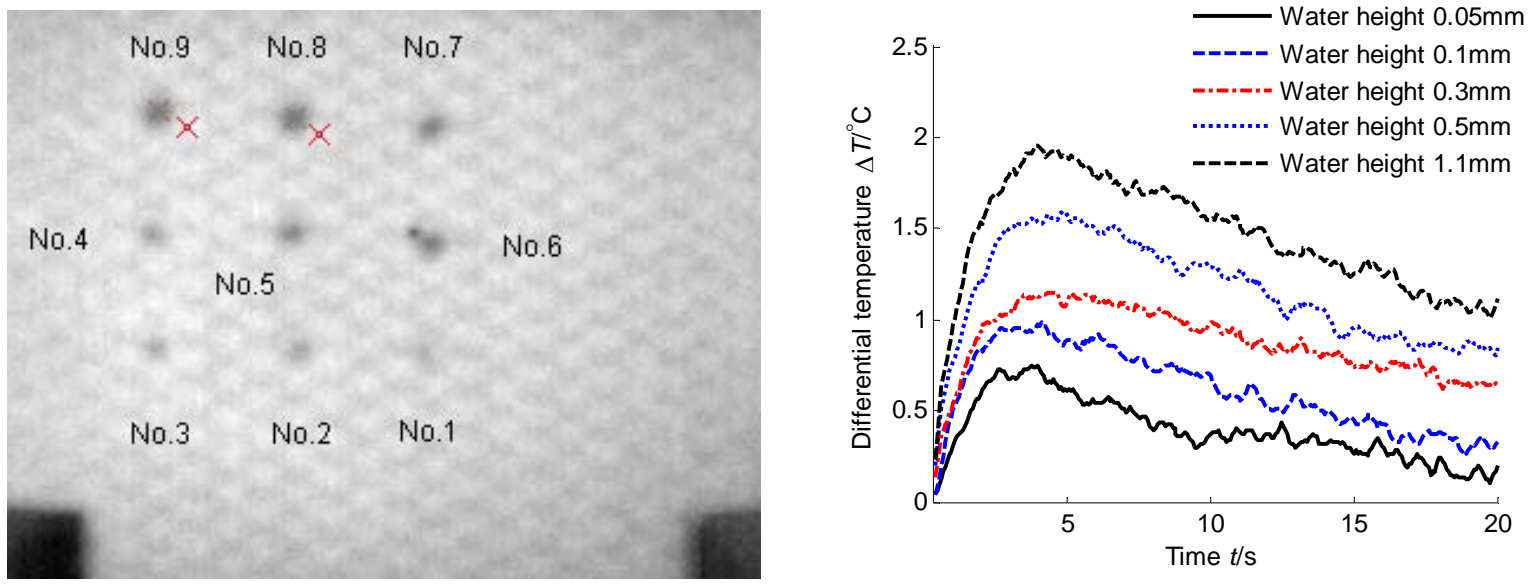

Fig.15. Excess temperature image in near water

Fig.16. Evolution of differential temperature in near water

testing of the specimen $(t=3.03 \mathrm{~s})$

testing of the specimen (after 5 point moving averaging)

To calculate the information parameters such as the differential temperature, maximum differential temperature and maximum contrast, a pair of points $(3 \times 3$ pixel size) is selected in each water area and its near sound area, as shown in Fig.15. The representative differential temperature curves of the specimen are shown in Fig.16. The maximum differential temperature and maximum contrast versus water height of the specimen are shown in Fig.17 and Fig.18 respectively. The quadratic equation Eq.(5) provides an excellent fit to the experimental maximum differential temperature vs. water height in Fig.17:

$$
\Delta T=-0.9218 h^{2}+2.1702 h+0.6907
$$

The quadratic equation Eq.(6) provides an excellent fit to the experimental maximum contrast vs. water height:

$$
C_{\mathrm{m}}=-0.2338 h^{2}+0.5107 h+0.0377
$$

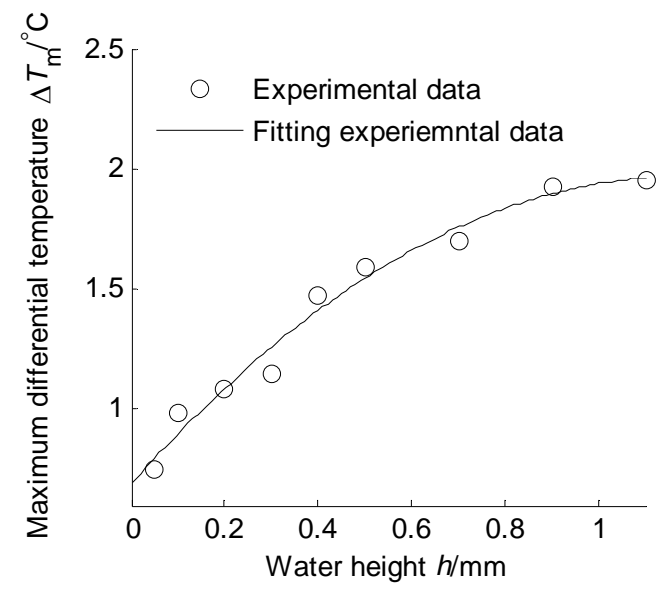

Fig.17. Maximum differential temperature vs. water height of the specimen

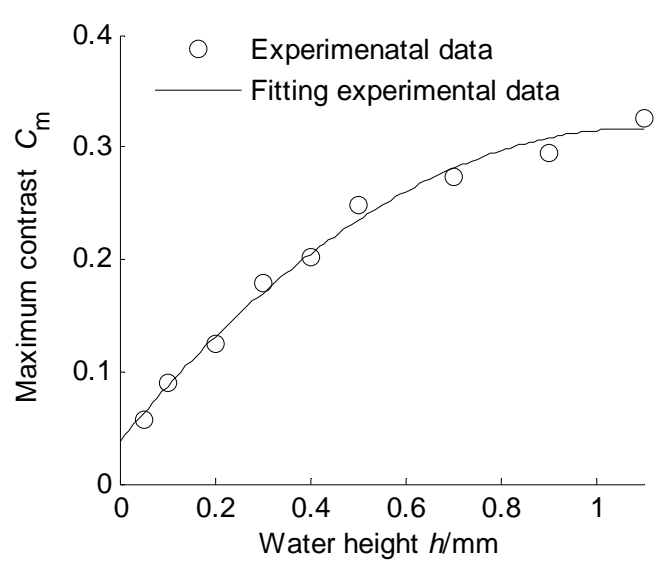

Fig.18. Maximum contrast vs. water height of the specimen 
The comparison of experimental data and simulation data are shown in Fig.19 and Fig.20. The change and tendency of the experimental data are basically the same as the corresponding simulation results. Because the differential temperature is proportional to the energy density absorbed, and the energy density in experiment is different from that in simulation, the differential temperature data is normalized by the value at $h=0.9 \mathrm{~mm}$ before the comparison. The experimental maximum contrast is smaller than the corresponding simulation result, this should attribute to two causes: the first is the modeling error that, the parameters of the specimen are different from the model's, and in the experiment, the little water gathers as a half ball instead of an uniform layer in the model; the second is the experimental error that various disturbing factors such as the location error of the center in water areas, uneven heating and nonuniform emissivity, etc., exist in the experiment.

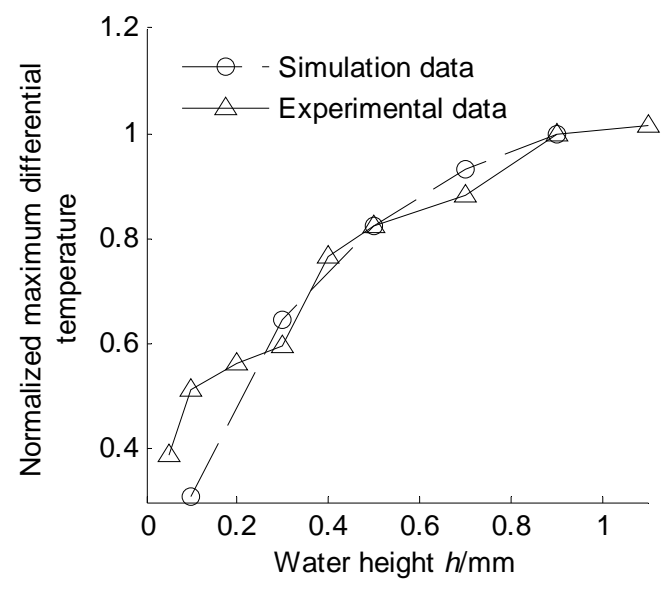

Fig.19. Comparison of normalized maximum differential temperature vs. water height for experiment and simulation

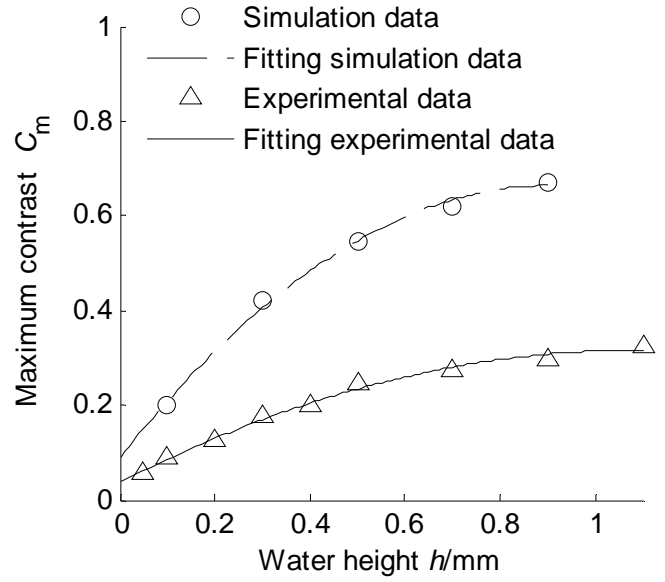

Fig.20. Comparison of maximum contrast vs. water height for experiment and simulation

\section{Conclusions}

(1) For any of the three testing approaches, if the water height is different, the excess temperature evolution will be different. The water height higher, the maximum differential temperature and maximum contrast become higher. The change tendency of the maximum contrast vs. water height is the same as the maximum differential temperature vs. water height.

(2) In near water testing, there is a non-linear relationship between the maximum differential temperature and water height, and the maximum differential temperature will tend to a saturation value.

(3) In vertical testing, the maximum differential temperature is almost a linear function of water height when the equivalent water height is less than $1 \mathrm{~mm}$.

(4) In far water testing, the air gap thickness can be used to judge if this testing is practicable. For the structure in this paper, if the total thickness of the concerned face panel and adhesive layer is more than $1 \mathrm{~mm}$, the energy density is less than $6000 \mathrm{~J} \cdot \mathrm{m}^{-2}$ and the air gap thickness is more than $3.4 \mathrm{~mm}$, the far water testing cannot be used.

(5) When water content is constant, the near water testing is most sensitive and has the best effect, the vertical testing takes the second place, and the far water testing has the worst effect.

(6) For the near water testing, the following conclusions exist: the maximum differential temperature decreases as the face panel thickness increasing, and the maximum differential temperature is almost inversely proportional to the face panel thickness; the maximum contrast also decreases as the face panel thickness increasing, and the maximum contrast is nearly a linear function of face panel thickness; the peak time of differential temperature is postponed when the face panel thickness increases, and the peak time is a linear function of the face panel thickness approximately.

(7) The simulation results of PT for detecting water ingress in honeycomb panels had been partially proved by the experiment of near water testing which indicated that the theoretical results are well comparable to the experimental results, and the change and tendency of the theoretical information parameters are basically the same as the corresponding experimental data. More experiment is needed to prove the theoretical predictions for the far water testing and vertical testing later. The conclusions of this paper provide a useful reference for the nondestructive testing and evaluation of water ingress in composite honeycomb panels.

\section{REFERENCES}

[1] Zauner G., Mayr G., Hendorfer G., "Comparative defect evaluation of aircraft components by active thermography". In: Niel K. S., Fofi D., Proc. of SPIE-IS\&T Electronic Imaging, Vol.7251, 72510J: pp.1-9, SPIE, 2009. 
[2] Ibarra-Castanedo C, Genest M, Guibert S, et al, "Inspection of aerospace materials by pulsed thermography, lock-in thermography and vibrothermography: A comparative study". In: Knettel K. M., Vavilov V. P., Miles J. J., Proc SPIE Thermosense XXIX, Bellingham, WA: SPIE, Vol.6541, 654116: pp.1-9, 2007.

[3] Garniera C., Pastorb M. L., Eymab F., et al, "The detection of aeronautical defects in situ on composite structures using non-destructive testing". Composite Structures, Vol.93(5), pp.1328-1336, 2011.

[4] Perez I., Davis W. R., Kulowitch P., "Thermographic modeling of water entrapment", In: Proc SPIE, Thermosense XXI, Orlando, Florida. pp.32-39, 1999.

[5] Anon, "Airbus adopts infrared thermography for in-service inspection". Insight, Vol.36(10), pp.774-775, 1994.

[6] Meng Tiejun, "Detecting water in honeycomb of Airbus's aircraft elevator in situ by infrared NDT", In: Proc of the 11th Annual Conference on Nondestructive Testing in Shaanxi Province, Hanzhong(Shaanxi), pp.42-46,14, 2008. (in Chinese)

[7] Vavilov V., Klimov A., Nesteruk D., Shiryaev V., "Detecting water in aviation honeycomb structures by using transient IR thermographic NDT", In: Cramer K., Maldague X., Proc SPIE, Thermosense XXV. Bellingham, WA: SPIE, Vol.5073, pp.345-355, 2003.

[8] Vavilov V., Nesteruk D., "Evaluating water content in aviation honeycomb panels by transient IR thermography", In: Peacock G. R., Burleigh D. D., Miles J. J., Proc SPIE, Thermosense XXVII, Bellingham, WA: SPIE, Vol.5782, pp.411-417, 2005.

[9] Vavilov V., Klimov A., Antoshkin S., et al, "Thermographic inspection to detect water ingress in composite sandwich panels in airplanes". NDT World, Vol.20(2), pp.11-12, 2003. (in Russian)

[10] Saarimaki E., Ylinen P., "An investigation of non-destructive thermographic inspection exploiting phase transition of water for moisture detection in aircraft structures". In: Proc. of QIRT 2008, Krakow(Poland), 2008.

[11] Zhao Shibin, Zhang Cunlin, Wu Naiming, "Pulsed thermography detection of water and hydraulic oil intrusion in the honeycomb sandwich structure composite". In: Puschell J. J., Chu Junhao, Gong Haimei, et al, Proc SPIE International Symposium on Photoelectronic Detection and Imaging 2011: Advances in Infrared Imaging and Applications, Bellingham, WA: SPIE, Vol.8193: 819337:pp.1-8, 2011. 